\title{
Parâmetros hematológicos de bezerros leiteiros mestiços do nascimento aos 15 dias
}

\section{de idade}

\author{
Hematological parameters of mixed dairy dairy birthers from birth to 15 days of age \\ Parámetros hematológicos de terneros mestizos lecheros desde el nacimiento hasta los 15 días de \\ edad
}

Recebido: 07/03/2021 | Revisado: 14/03/2021 | Aceito: 09/06/2021 | Publicado: 21/06/2021

\author{
Patrícia de Queiroz Ribeiro Mattos \\ ORCID: https://orcid.org/0000-0001-9708-7410 \\ Universidade Federal Rural do Rio de Janeiro, Brasil \\ E-mail: pqueirozvet@yahoo.com.br \\ Rosângela Antunes Terra \\ ORCID: https://orcid.org/0000-0001-6740-5393 \\ Instituto Federal de Educação, Ciência e Tecnologia do Rio de Janeiro, Brasil \\ E-mail: rosangelaterra@ifrj.edu.br \\ Rita de Cássia Campbell Machado Botteon \\ ORCID: https://orcid.org/0000-0001-9837-7532 \\ Universidade Federal Rural do Rio de Janeiro, Brasil \\ E-mail: ritabotteon@gmail.com
}

\begin{abstract}
Resumo
Ao nascer bezerros possuem características metabólicas semelhantes aos monogástricos e os parâmetros hematológicos variam conforme a fase de desenvolvimento. Ainda são poucos os dados publicados que permitem concluir com segurança se alterações hematológicas de neonatos são decorrentes de enfermidades ou devido à processos fisiológicos. Assim, este estudo teve como objetivo avaliar a hematologia de 15 bezerros leiteiros mestiços do nascimento aos 15 dias de idade, amamentados pela mãe no primeiro momento pós-parto e, posteriormente, com colostro ou leite de manhã e à tarde, por meio de baldes.A partir do terceiro dia de vida foi ofertado alimento sólido (volumoso (feno) e concentrado). Em até 2 horas após o nascimento, com12 horas, e nos dias dois, três, cinco, sete, 10, 12 e 15 foram colhidas amostras de sangue em frascos com EDTA para a realização e hemograma. Houve aumento $(\mathrm{p} \geq 0,05)$ na contagem de hemácias entre o nascimento e 12 horas e estabilização a seguir. O VCM teve redução do nascimento aos 15 dias. O VG ficou próximo do limite da normalidade. Na média as concentrações de fibrinogênio foram normais ao longo dos 15 dias, sendo os valores mais elevados entre três e sete dias $(\mathrm{p}<0,01)$. As menores e maiores médias de PPT foram 6,28 $\pm 0,78 \mathrm{~g} / \mathrm{dL}$ aos cinco dias, e 6,65 $\pm 1,03$ aos 15 dias. Leucócitos e neutrófilos segmentados foram ligeiramente elevados e os linfócitos diminuídos 12 horas após o nascimento. Em seguida, os valores inverteram-se. Conclui-se que as variações observadas são atribuídas a mudanças fisiológicas, sendo a influência do fator etário significativa.
\end{abstract}

Palavras-chave: Ruminantes; Neonatos; Hemograma.

\begin{abstract}
When calves are born, they have metabolic characteristics similar to monogastric ones and hematological parameters vary according to the stage of development. There are still few published data that allow us to safely conclude whether hematological changes in neonates are due to illnesses or due to physiological processes. Thus, this study aimed to evaluate the hematology of 15 crossbred dairy calves from birth to 15 days of age, breastfed by the mother in the first postpartum moment and, later, with colostrum or milk in the morning and in the afternoon, through buckets .From the third day of life, solid food (roughage (hay) and concentrate) was offered. Within 2 hours after birth, with 12 hours, and on days two, three, five, seven, 10, 12 and 15, blood samples were collected in flasks with EDTA for the performance and blood count. There was an increase $(p \geq 0.05)$ in the red blood cell count between birth and 12 hours and stabilization thereafter. VCM had a reduction in birth at 15 days. The VG was close to the normal limit. On average, fibrinogen concentrations were normal over the 15 days, with the highest values between three and seven days ( $\mathrm{p}<0.01$ ). The lowest and highest PPT averages were $6.28 \pm 0.78 \mathrm{~g} / \mathrm{dL}$ at five days, and $6.65 \pm 1.03$ at 15 days. Leukocytes and segmented neutrophils were slightly elevated and lymphocytes decreased 12 hours after birth. Then, the values reversed. It is concluded that the observed variations are attributed to physiological changes, with the influence of the age factor being significant.
\end{abstract}

Keywords: Ruminants; Neonates; Blood count. 


\begin{abstract}
Resumen
Al nacer los terneros tienen características metabólicas similares a los parámetros monogástricos y hematológicos varían según la fase de desarrollo. Todavía hay pocos datos publicados para concluir con seguridad si las alteraciones hematológicas de los neonatos se deben a enfermedades o debido a procesos fisiológicos. Así, este estudio tenía como objetivo evaluar la hematología de 15 terneros lácteos de raza cruzada desde el nacimiento hasta los 15 días de edad, amamantados por la madre en el primer momento posparto y, posteriormente, con calostro o leche por la mañana y por la tarde, a través de cubos. Desde el tercer día de vida, se ofrecieron alimentos sólidos (voluminosos (heno) y concentrados. Dentro de las 2 horas posteriores al nacimiento, a las 12 horas, y los días dos, tres, cinco, siete, 10, 12 y 15, se recogieron muestras de sangre en viales con EDTA para su realización y recuento de sangre. Hubo un aumento $(\mathrm{p} \geq 0.05)$ en el recuento de glóbulos rojos entre el nacimiento y las 12 horas y la estabilización a partir de entonces. El MVC tuvo una reducción en el nacimiento a los 15 días. El VG estaba cerca del límite de normalidad. En promedio, las concentraciones de fibrinógenos fueron normales durante los 15 días, con los valores más altos entre tres y siete días ( $\mathrm{p}<0.01$ ). El SPP medio más bajo y más alto fue de 6,28 $\pm 0,78 \mathrm{~g} / \mathrm{dL}$ a cinco días, y 6,65 1 1,03 a 15 días. Los leucocitos y los neutrófilos segmentados fueron ligeramente elevados y los linfocitos disminuyeron 12 horas después del nacimiento. A continuación, los valores se invirtieron. Se concluye que las variaciones observadas se atribuyen a cambios fisiológicos, y la influencia del factor de edad es significativa. Palabras clave: Rumiantes; Reciénnacidos; Conteo de glóbulos.
\end{abstract}

\title{
1. Introdução
}

O hemograma, composto por avaliações qualitativas e quantitativas dos elementos sanguíneos permite, de maneira fácil, a obtenção de informações valiosas sobre a saúde e adaptação aos desafios ambientais (Hulbert \& Moisá, 2016). Além das características espécies-específicas, as adaptações fisiológicas, podem influenciar os constituintes do sangue e a interpretação dos resultados do hemograma (Thrall et al., 2015).

A interpretação do hemograma de bezerros neonatos requer a adoção de intervalos de referência consistentes com a idade (Brun-Hansen et al., 2006; Novo et al., 2015; Panousis et al., 2018), sendo não recomendável para estes animais, utilizar valores estabelecidos para adultos. Os ruminantes recém-nascidos diferem dos adultos em vários aspectos e o conhecimento dos valores de referência dos animais sadios, bem como dos fatores que causam variações destes parâmetros é fundamental para formular planos diagnósticos e direcionar o tratamento de diferentes enfermidades (Hulbert \& Moisá, 2016). Os valores para bezerros disponíveis na literatura em geral abrangem uma faixa etária ampla (dias, semanas ou meses) e condições de criação diversas.

No presente estudo (aprovado pela Comissão de Ética no Uso de Animais em Pesquisa /UFRRJ, processo 0058-062019), avaliou-se os valores dos constituintes do hemograma de bezerros mestiços, do nascimento aos 15 dias de vida, mantidos em condições que representam o manejo utilizado na maioria das criações brasileiras.

\section{Metodologia}

Este estudo caracteriza-se pelo método quali-quanti nos quais os resultados numéricos são complementados por resultados qualitativos (Pereira et al., 2018). Os resultados foram apresentados como média e desvio padrão, onde os que apresentavam distribuição normal foram comparados por meio de análise de variância multivariada e para determinação da ocorrência de diferenças fora utilizado teste de Tukey a $95 \%$ de significância $(\mathrm{p} \leq 0,05)$.

Foram avaliados 15 bezerros mestiços holandês-zebu, do nascimento aos 15 dias de idade do setor de Bovinocultura no Instituto Federal do Rio de Janeiro, Campus Pinheiral - Rio de Janeiro, entre junho de 2019 e janeiro de 2020.

Duas semanas antes da data prevista para a parição, as vacas foram alocadas em piquete maternidade e monitoradas quanto aos sinais de parto, o qual, sempre que possível, foi acompanhado por funcionários.

Ao nascimento os bezerros foram mantidos com as mães por até 12 horas, e em seguida apartados, identificados, pesados, e foi realizada a desinfecção do umbigo (imersão em solução iodada $7 \%$ por 60 segundos, repetida por mais dois 
dias). Após, dois litros de colostro da primeira ordenha das respectivas mães foram fornecidos em mamadeira e os bezerros alojados em baias individuais, em galpão de alvenaria coberto com telhas de barro e piso de madeira. As baias foram limpas diariamente com vassoura e jatos de água.

Nos dois dias seguintes ao nascimento, os bezerros receberam leite de transição das respectivas mães, pela manhã e à tarde em volume final de $10 \%$ do peso vivo/dia conforme Azevedo et al. (2013). Nos dias subsequentes foram alimentados com leite integral in natura, dois litros pela manhã e dois à tarde, por meio de baldes com bicos. Água foi disponibilizada à vontade, em baldes de metal, desde o primeiro dia. Volumoso e concentrado comercial foram fornecidos para consumo voluntário a partir do terceiro dia.

Os animais foram inspecionados pela manhã, quanto à condição corporal e sinais de enfermidades, com ênfase no sistema digestivo (apetite, sucção e observação das fezes). Em seguida foram aferidos os parâmetros vitais (temperatura, frequências respiratória e cardíaca, tempo de preenchimento capilar e turgor cutâneo). Animais com alterações nesses parâmetros foram avaliados detalhadamente conforme Feitosa (2014). Aqueles que apresentaram sinais de enfermidades sistêmicas, desidratação, depressão ou que necessitaram de intervenção terapêutica foram excluídos.

Amostras de 5,0 mL de sangue foram obtidas por punção da jugular, após assepsia local, utilizando-se agulhas 40x12 mm, em frascos com EDTA a 10\%, logo após a identificação do nascimento (D0 - até duas horas), 12 horas após (12H),e a seguir $\operatorname{aos} 2,3,5,7,10,12$ e 15 dias, pela manhã, imediatamente antes de cada refeição. A contenção foi realizada manualmente, por um auxiliar, de maneira tranquila.

Imediatamente após a colheita foram determinadoso volume globular (VG - \%) pela técnica do microhematócrito, a

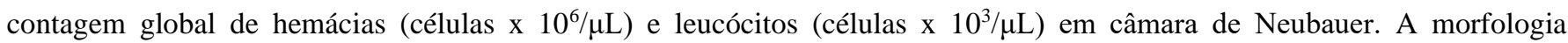
eritrocitária e a leucometria específica (percentual de neutrófilos, linfócitos, eosinófilos, monócitos e basófilos) foram avaliadas em esfregaço sanguíneo corado com corante hematológico rápido, com auxílio de microscópio óptico, aumento de 1000 vezes (Jain, 1993).

\section{Resultados e Discussão}

Os constituintes do eritrograma representados na tabela 1 apresentaram uma variação discreta do $2^{\circ}$ ao $15^{\circ}$ dia do nascimento.

As menores médias da contagem de hemácias ocorreram ao nascimento $\left(8,69 \pm 1,46 \times 10^{6} / \mu \mathrm{L}\right)$ e as maiores 12 horas após $\left(10,02 \pm 2,22 \times 10^{6} / \mu \mathrm{L}\right)$, sem diferença significativa $(\mathrm{p} \geq 0,05)$ entre os momentos. Essa variação ficou dentro da faixa de normalidade estabelecida por Feldman et al. (2000) e Kaneko (1997), para bovinos adultos, utilizados pela maioria dos laboratórios de patologia clínica veterinária. 
Tabela 1: Médias e desvio padrão da contagem global de hemácias, volume globular evolume corpuscular médio (VCM) em bezerros leiteiros mestiços, ao nascimento (até 2 horas - D0), 12 horas após o nascimento, aos 2, 3, 5, 7, 10, 12 e 15 dias de idade.

\begin{tabular}{cccc}
\hline & Hemácias & Volume Globular & VCM \\
Referência & $\left(6\right.$ a $\left.12 \times 10^{6} / \mu \mathrm{L}\right)$ & $(32$ a $48 \%)$ & $(34$ a $58 \mathrm{fL})$ \\
\hline D 0 & $8,69 \pm 1,46$ & $37,6 \pm 9,8$ & $44,1 \pm 8,5$ \\
12 h & $10,02 \pm 2,22$ & $37,9 \pm 10,2$ & $41,4 \pm 6,2$ \\
2 D & $9,21 \pm 2,98$ & $36,4 \pm 9,7$ & $42,0 \pm 8,3$ \\
3 D & $9,96 \pm 2,27$ & $35,8 \pm 10,1$ & $39,1 \pm 6,2$ \\
5 D & $9,79 \pm 1,19$ & $37,3 \pm 7,4$ & $38,4 \pm 5,8$ \\
7 D & $8,97 \pm 2,23$ & $36 \pm 6,0$ & $40,3 \pm 6,2$ \\
10 D & $9,72 \pm 2,14$ & $34,5 \pm 9,3$ & $36,9 \pm 6,4$ \\
12 D & $9,56 \pm 2,08$ & $34,8 \pm 9,3$ & $36,2 \pm 6,0$ \\
15D & $8,93 \pm 1,90$ & $32,6 \pm 9,2$ & $36,3 \pm 5,9$ \\
\hline Valor de p & 0,8105 & 0,9169 & 0,1256 \\
\hline
\end{tabular}

** Diferença significativa a 95\% de confiança em relação aos demais dias. Referência: Feldman et al. (2000); *Kaneko et al. (2008).

Contagem mais baixa de hemácias ao nascimento difere de outros estudos (Pinna et al., 2001; Rengifo et al., 2010; Benesi et al., 2012; Rocha et al., 2013, Novo et al., 2015; Baccili et al., 2018; Franciosi et al., 2018) provavelmente em função do momento de coleta da amostra, que é bastante variável entre os estudos.

Pinna et al. (2001) e Rengifo et al. (2010), em condições de manejo semelhantes, obtiveram para bezerros leiteiros mestiços, respectivamente de 1 a 7 e de 1 a 30 dias de idade, hematimetria e VG menores que os encontrados no presente estudo até os 15 dias. O valor de VG encontrado por Rengifo et al. (2010) neste período ficou abaixo do valor de referência.

Também Rocha et al. (2013) observaram variações discretas e valores de hemácias decrescentes do nascimento ao sétimo dia, porém normais para a espécie. Franciosi et al. (2018) verificaram um decréscimo progressivo da hematimetria até o $20^{\circ}$ dia. Novo et al. (2015) e Bacccili et al. (2018) em bezerros da raça holandesa encontraram VG mais elevado ao nascimento e valores decrescentes até seis e quatro dias de vida, respectivamente. Benesi et al. (2012) realizaram um estudo hematológico mais amplo em 300 bezerros da raça holandesa, avaliados em intervalos de horas ou dias. Os valores reportados foram inferiores aos do presente estudo quanto à hematimetria, VG e VCM, em todos os momentos.

As médias da contagem de hemácias e VG descritas por Rocha et al. (2013), Calixto (2013), Novo et al. (2015) e Fioravanti et al. (2016) foram próximas das encontradas no presente estudo, contudo Fioravanti et al. (2016) avaliaram bezerros de zero a 90 dias, sem distinção de idade, diferentemente do presente estudo.

A contagem de hemácias registrada por Baccili et al. (2018) em bezerras da raça Holandesa ao nascimento $(8,5 \pm 1,3$ x $\left.10^{6} / \mu \mathrm{L}\right)$ foram próximas, porém o $\mathrm{VG}(39 \pm 6 \%)$ e o VCM $(46 \pm 1 \mathrm{fL})$ foram mais elevados, e igualmente ao presente estudo, decrescentes nos primeiros dias de vida.

A redução gradual no VCM após o nascimento foi também reportada por Novo et al. (2015) e Baccili et al. (2018). Outros estudos com raças diferentes (Birgel Junior, 1991; Bomfim, 1995; Marçal et al., 1995; Távora, 1997; Gonçalves et al., 2001; Paula Neto, 2004) evidenciaram aumento do VCM, sem distinção de idade.

Em função da ingestão de colostro, que além de sua função primordial para aquisição de imunidade passiva, pela absorção de imunoglobulinas, também é importante do ponto de vista da nutrição, hidratação e equilíbrio metabólico dos bezerros neonatos (Garry et al., 1993) é provável haver uma redução nos valores dos constituintes do eritrogramanas primeiras horas de vida (Souza et al., 2018), o que não foi evidenciado no presente estudo, em que os parâmetros 
eritrocitários foram maiores nos bezerros com 12 horas de nascidos.

Falhas de manejo no período de colostro podem levar à hemoconcentração, que resulta em aumento do VG e contagens mais elevadas de hemácias nos primeiros dias de vida, sendo esta uma possível causa para o aumento do VG e hematimetria no presente estudo. Embora a água seja um nutriente vital, existem poucos estudos sobre a prevalência de desidratação em bezerros neonatos sem diarreia e sua carência tem sido mal descrita nos modelos de necessidade de nutrientes (Wickramasinghe et al., 2019).

Renaud et al. (2018) identificaram que $12 \%$ dos bezerros alimentados exclusivamente com leite, apresentavam sinais clínicos de desidratação. Como demonstrado por Muller (2017), a baixa ingestão de leite está associada à mortalidade de bezerros mediada por desidratação, sendo este um importante aspecto a ser considerado no manejo dos neonatos.

Estudo realizado pelo sistema de monitoramento de saúde animal do Departamento de Agricultura dos Estados Unidos (USDA, 2016) demonstrou que os produtores de leite aguardam, em média, 17 dias para oferecer água aos bezerros presumindo que causaria diarreia (Kertz et al., 2017). É possível que atitude semelhante ocorra no Brasil e estudos realizados em condições reais de campo podem resultar em valores diferentes dos constituintes do eritrograma em relação à estudos em animais com água fornecida para consumo voluntário, como no presente estudo.

A variação decrescente do VG, do nascimento aos 15 dias e do VCM aponta para o desenvolvimento de anemia. Wise et al. (1947) e Tennant et al. (1974) reportaram que o declínio do VCM em bezerros neonatos é um processo fisiológico e conforme Jain (1993) coincide a substituição dos eritrócitos e hemoglobina fetais por células de adultos (menores). O declínio observado é consistente com observações de Golbeck et al. (2019) sobre o desenvolvimento de anemia microcítica leve, que, se associada à hipocromia caracteriza deficiência de ferro, sendo esperada anemia em 20 a $30 \%$ dos bezerros.

Dentre 164 bezerros de até quatro meses, de 25 rebanhos Holstein, avaliados por Ramin et al. (1975), 17,7\% apresentaram anemia leve não relacionada à deficiência de ferro. Noventa dias foi a idade crítica para ocorrência de anemia, portanto superior ao período de avaliação no presente estudo. No Brasil, Benesi et al. (2019) identificaram anemia em 14,7\% dos bezerros da raça Holandesa no primeiro mês de vida, sendo sugerida uma possível etiologia ferropriva, na maioria dos casos.

Pelos dados demonstrados na tabela 02, observam-se após o nascimento, contagens ligeiramente elevadas de leucócitos totais e neutrófilos segmentados, ao mesmo tempo em que os linfócitos estavam diminuídos. Foi constatado um aumento gradual de linfócitos enquanto que os neutrófilos diminuíram com o aumento da idade. As contagens de leucócitos totais, neutrófilos segmentados e linfócitos se normalizaram 12 horas após o nascimento, mantendo-se normais nos dias seguintes. 
Tabela 2: Médias e desvio padrão de leucócitos totais e contagem diferencial de neutrófilos, linfócitos, monócitos, basófilos e eosinófilos em bezerros leiteiros mestiços até 2 horas após o nascimento (D0), 12 horas e de dois aos 15 dias de idade.

\begin{tabular}{ccccccc}
\hline & Leu totais & Segmentados & Linfócitos & Monócitos & Basófilos & Eosinófilos \\
\hline D0 & $12.077 \pm 3.574$ & $50,3 \pm 11,0$ & $42,8 \pm 9,1$ & $2,4 \pm 1,9$ & $1,0 \pm 1,5$ & $2,0 \pm 2,1$ \\
12h & $10.765 \pm 3.219$ & $40,9 \pm 10,3$ & $52,0 \pm 9,9$ & $3,1 \pm 2,1$ & $1,1 \pm 1,2$ & $1,6 \pm 1,8$ \\
2D & $10.523 \pm 2.905$ & $43,3 \pm 12,2$ & $52,8 \pm 12,7$ & $4,0 \pm 2,5$ & $1,2 \pm 1,9$ & $1,6 \pm 1,6$ \\
3D & $10.150 \pm 3.353$ & $40,2 \pm 9,2$ & $53,3 \pm 9,0$ & $3,5 \pm 1,8$ & $1,2 \pm 1,7$ & $1,7 \pm 1,5$ \\
5D & $11.564 \pm 2.766$ & $40,8 \pm 9,0$ & $52,3 \pm 11,3$ & $4,2 \pm 3,3$ & $1,3 \pm 1,9$ & $1,0 \pm 1,2$ \\
7D & $11.385 \pm 3.760$ & $37,1 \pm 13,0$ & $55,6 \pm 14,3$ & $3,85 \pm 2,3$ & $1,7 \pm 1,9$ & $1,8 \pm 2,0$ \\
10D & $11.469 \pm 4152$ & $42,2 \pm 10,9$ & $49,6 \pm 13,4$ & $4,3 \pm 3,1$ & $1,5 \pm 2,2$ & $1,9 \pm 1,9$ \\
12D & $12.018 \pm 2999$ & $41,6 \pm 6,6$ & $52,8 \pm 9,6$ & $2,9 \pm 2,2$ & $1,0 \pm 1,4$ & $1,5 \pm 1,6$ \\
15D & $11.627 \pm 4448$ & $34,9 \pm 9,0$ & $57,5 \pm 5,8$ & $3,4 \pm 3,4$ & $1,9 \pm 1,5$ & $1,9 \pm 1,7$ \\
\hline Referência* & $4 \mathrm{a} 12 \times 10^{3} / \mu \mathrm{L}$ & $15-45 \%$ & $45-75 \%$ & $2-7 \%$ & $0-2 \%$ & $2-20 \%$ \\
\hline Valor de p & 0,8864 & 0,0705 & 0,1429 & 0,6825 & 0,9259 & 0,9414 \\
\hline
\end{tabular}

*Feldman; Zinkl e Jain (2000).

De 12 horas aos 15 dias do nascimento, os neutrófilos segmentados diminuíram enquanto os linfócitos aumentaram, mantendo-se ambos dentro do intervalo de referência estabelecido para bovinos adultos, mesmo com variações nos resultados observados por diferentes autores (Jain, 1993; Jones \& Allison, 2007; Kaneko et al., 2008; Weiss \& Wardrop, 2010).

Contagens de leucócitos e neutrófilos ligeiramente elevadas ao nascimento são consistentes com observações de Knowles et al. (2000) quando da comparação entre neonatos e bovinos adultos. Porém, outros autores indicaram contagens de bezerros neonatos dentro dos limites de referência (Mohri et al. 2007) ou menores (Jain, 1993).

Conforme Jain (1993) é comum ocorrer um pequeno número de neutrófilos imaturos na circulação de bezerros neonatos e aumento da relação neutrófilos:linfócitos (N:L), provavelmente refletindo uma resposta fisiológica ao cortisol sérico aumentado, porém esta relação declina e se inverte nas primeiras semanas.

No bezerro neonato, de forma similar ao adulto, o estresse pode desencadear uma série de reações fisiológicas importantes e induzir a resultados diferenciados no hemograma (Aguayo-Ulloa et al., 2014; Cafazzo et al., 2014; Alm et al., 2016; Hulbert \& Moisá, 2016). O eixo hipotálamo-hipofisário adrenal pode ser ativado por marcadores inflamatórios e estresse (Fleshner, 2013). Em resposta, a secreção de glicocorticoides fornece sinais pró e anti-inflamatórios aos tecidos imunológicos e estimula a liberação de neutrófilos e células da medula óssea para a circulação (Schalm, 2010).

Especialmente o leucograma é influenciado pela atividade adrenocortical, referenciado como leucograma de estresse, caracterizado por leucocitose primariamente por neutrofilia com linfopenia e redução de eosinófilos (Cafazzo et al., 2014; Dickson, 1996). De acordo com Eberhart e Patt (1971), Jacob et al. (2001) e Benesi (1992) a concentração de cortisol plasmático é elevada em vacas no dia do parto e em bezerros ao nascimento, e ocorre um grande declínio nos primeiros dias, acompanhada da redução de neutrófilos, elevação de linfócitos, monócitos e eosinófilos no sangue periférico (Nathanielsz et al., 1980).

Conforme Taylor (2000), a relação N:L deve diminuir de 2,8 ao nascimento para 0,5 aos 5 dias de idade, permanecendo constante até a fase adulta. No presente estudo esta relação variou de 1,2 ao nascimento a 0,812 horas após e 0,6 aos 15 dias de idade, sugerindo baixos níveis de cortisol adequação do manejo a que os animais foram submetidos (Paes et al., 2012). A inversão na proporção N:L imediatamente após o nascimento difere de outros autores que registraram a inversão mais tardiamente, aos 15 dias de vida (Costa et al., 2008; Fagliari et al. 1998; Biondo et al. 1998; Peixoto et al., 2002).

Benesi et al. (2012) observaram valores máximos nas primeiras oito horas para leucócitos, segmentados e neutrófilos 
totais e entre 9 a 16 horas pós-nascimento para os neutrófilos bastonetes. Para os linfócitos totais os valores máximos ocorreram entre 21 e 30 dias, diferindo do presente estudo.

Novo et al. (2015) demostraram que o primeiro mês de vida é um período de adaptação hematológica com variações de hemocomponentes, caracterizadas por hemoconcentração e perfil leucocitário compatível com a resposta de glicocorticóides até o $4^{\circ}$ dia de vida e proporção de neutrófilos:linfócitos >1,0 nas primeiras 48 horas de vida.

\section{Considerações Finais}

Variações hematológicas em bezerros neonatos sadios são atribuídas à mudanças fisiológicas inerentes ao desenvolvimento, sendo a influência do fator etário muito significativo.

Os valores obtidos podem ser utilizados como referenciais para bezerros mestiços neonatos criados em condições similares ao presente estudo.

Frente ao exposto, consideramos importante a continuidade de estudos sobre avaliação da hematologia sérica por constituir importante ferramenta de monitoramento e avaliação de bezerros neonatos, visando a oferecer subsídios ao diagnóstico de enfermidades comuns neste período.

\section{Referências}

Aguayo-Ulloa, L. A., Villarroel, M., Pascual-Alonso, M., Miranda-De La Lama, G. C. \& María, G. A. (2014). Finishing feed lot lambs in enriched pens using feeder ramps and straw and its influence on behavior and physiological welfare indicators. Journal of Veterinary Behavior, 9, 347-356.

Alm, M., Tauson, R., Holm, L., Wichman, A., Kalliokoski, O. \& Wall, H. (2016). Welfare indicators in laying hens in relation to nest exclusion. Poultry Science.95:1238-1247.

Azevedo, R. A., Araújo, L., Duarte, D. V. L., Cruz, M. S., Costa, S. F., Oliveira, N. J. F., Duarte, E. R. \& Geraseev, L. C. (2013). Desenvolvimento do trato digestivo de bezerros leiteiros criados em sistema de aleitamento fracionado. Pesquisa Veterinária Brasileira, 33, $931-936$.

Baccili, C. C., Oliveira, S. M. F. N., Costa, J. F. R., Massoco, C. O., Pozzi, C. R. \& Gomes, V. (2018). Desenvolvimento hematológico e imunológico do nascimento aos seis meses de idade em bezerros da raça Holandesa. Arquivo Brasileiro de Medicina Veterinária e Zootecnia, 70(6), $1823-1832$.

Benesi, F. J., Teixeira, C. M. C., Lisboa, J. A. N., Leal, M. L. R., Birgel Júnior, E. H., Bohland, E. \& Regina, M. S. (2012). Eritrograma de bezerras sadias, da raça Holandesa no primeiro mês de vida. Pesquisa Veterinária Brasileira, 32(4), 357-360.

Benesi, F. J., Lisbôa, J. A. N., Leal, M. L. R., Shecaira, C. L. \& Santos, R. B. (2019). Occurrence of anemia in Holstein calves in the first month after birth.Sêmina: Ciências Agrárias, 40(3), 1139-1144.

Biondo, A. W., Lopes, S. T. A., Kohayagawa, A., Takahira, R. K. \& Alencar, N. X. (1998). Hemograma de bovinos (Bosindicus) sadios da raça Nelore no primeiro mês de vida, criados no estado de São Paulo. Ciência Rural. 28(2), 251-256.

Birgel Jr., E. H. (1991). O hemograma de bovinos (Bostaurus, Linnaeus, 1758), da raça Jersey, criados no Estado de São Paulo. Dissertação (Mestrado em Patologia Bovina) - Faculdade de Medicina Veterinária e Zootecnia, Universidade de São Paulo, São Paulo.

Bomfim, S. R. M. (1995). Mielograma e hemograma em bezerros bubalinos (Bubalus bubalis), do nascimento até um ano de idade. Dissertação 58 (Mestrado em Clínica Veterinária) - Faculdade de Medicina Veterinária e Zootecnia, Universidade Estadual Paulista, Botucatu.

Boutzios, G. \& Kaltsas, G. (2000). Immune System Effects on the Endocrine System. L. J. De Groot, P. Beck-Peccoz, G. Chrousos. ed. MDText.com Inc., South Dartmouth, MA.

Brun-Hansen, H. C., Kampen, A. H. \& Lund, A. (2006). Hematologic values in calves during the first 6 months of life. Veterinary Clinical Pathology, 35(2), $182-187$.

Calixto, L. M. (2013). Influência etária e nutricional na hematologia de bezerros da raça Holandesa. Dissertação (Mestrado em Clínica Veterinária) Universidade Federal de Minas Gerais, UFMG.

Cafazzo, S., Maragliano, L., Bonanni, R., Scholl, F., Guarducci, M., Scarcella, R., Dipaolo, M., Pontier, D., Lai, O., Carlevaro, F., Bucci, E., Cerini, N., Carlevaro, L., Alfieri, L., Fantini, C. \& Natoli, E. (2014). Behavioural and physiological indicators of shelter dogs' welfare: reflections on the no-kill policy on free-ranging dogs in Italy revisited on the basis of 15 years of implementation. Physiology \& Behavior.133, 223-229.

Costa, M. C., Flaiban, K. K. M. C., Coneglian, M. M., Feitosa, F. L. F., Balarin, M. R. S. \& Lisboa, J. A. N. (2008). Transferência de imunidade passiva em bezerros das raças nelore e limousin nos primeiros quarto meses de vida. Pesquisa Veterinária Brasileira, 28(9), 410-416.

Dickson, W. M. (1996). Glândulas endócrinas. In: Swenson, M.J. \& Reece, W.O. Dukes fisiologia dos animais domésticos (11a ed.). Guanabara Koogan, cap.34, 596-598. 
Eberhart, R. J. \& Patt, J. J. A. (1971). Plasma cortisol concentrations in newborn calves.Journal of Veterinary Research, 32, 1921-1927.

Fagliari, J. J., Santana, A. E., Lucas, F. A., Campus Filho, E. \& Curi, P. R. (1998). Constituintes sanguíneos de bovinos lactentes, desmamados e adultos das raças Nelore (Bosindicus) e Holandesa (Bostaurus) e de bubalinos (Bubalusbubalis) da raça Murrah. Arquivo Brasileiro de Medicina Veterinária e Zootecnia, $33(3), 263-271$.

Feitosa, F. L. F. (2014). Semiologia veterinária: A arte do diagnóstico (3a ed.). Roca.

Feldman, B. F., Zinkl, J. G. \& Jain, N. C. (2000). Schalm's veterinary hematology (5a.ed). Lippincott Williams and Wilkins.

Fioravanti, M. C. S., Neto, P. J. B., Juliano, R. S., Nunes, A. C. B., Lobo, J. R., Borges, A. C., Sereno, J. R. \& Miguel, M. P. (2016). Valores hematológicos de bovinos sadios da raça curraleiro pé duro (Bostaurus): efeito da idade, sexo e gestação. Actas Ibero-Americana sem Conservación Animal - AICA, 7, 8-15.

Fleshner, M. (2013). Stress-evoked sterile inflammation, danger associated molecular patterns (DAMPs), microbial associated molecular patterns (MAMPs) and the inflammasome.Brain, Behavior, and Immunity, 27, 1-7.

Franciosi, C., Rocha, T. G. \& Fagliari, J. J. (2018). Parâmetros hematológicos e bioquímicos de bezerros neonatos da raça Holandesa tratados com ferro suplementar. Pesquisa Veterinária Brasileira, 38(2), 234-243.

Garry, F., Aldridge, B. \& Adams, R. (1993). Role of colostral transfer in neonatal calf management: currentconcepts in diagnosis. Compendium on Continuing Education for the Practicing Veterinarian, 159(8), 1167-1176.

Golbeck, L., Cohrs, I., Scheu, T. \& Grünberg, W. (2019). Changes of the erythrocyte phenotype and blood biochemistry in dairy calves during the first ten weeks of age. Peers, e7248. Recuperado de https://doi.org/10.7717/peerj.7248

Gonçalves, R. C., Paes, P. R. O., Almeida, C. T., Fonteque, J. H., Lopes, R. S., Kuchembuck, M. R. G. \& Crocci, A. J. (2001). Influência da idade e sexo sobre o hemograma, proteínas séricas totais, albumina e globulina de bovinos sadios da raça Guzerá (Bosindicus). Veterinária Notícias, 7(1), 61-68.

Hulbert, L. E. \& Moisá, S. J. (2016). Stress, immunity, and the management of calves.Journal of Dairy Science, 99(4), 3199-3216.

Jacob, S. K., Ramnath, V., Philomina, P. T., Raghunandhanan, K. V. \& Kannan, A. (2001). Assessment of physiological stress in periparturient cows and neonatal calves.Indian Journal of Physiology and Pharmacology, 45(2), 233-238.

Jain, N. C. (1993). Essentials of veterinary hematology. Lea \& Febiger.

Jones, J. L. \& Allison, R. W. (2007). Evaluation of the ruminant complete blood cell count.The Veterinary Clinics of North America - Food Animal Practice, $23,377-402$.

Kaneko, J. J. \& Mills, R. (1970). Hematological and blood chemical observation in neonatal normal and porphyric calves in early life. Cornell Veterinary, $60(1), 52-60$.

Kaneko, J. J. (1997). Carbohidrate Metabolism and its disease. In: Kaneko, J. J. (1997). Clinical biochemistry of domestic animals (5a.ed., 45-81). Academic Press.

Kaneko, J. J., Harvey, J. W. \& Bruss, M. L. (2008). Clinical Biochemistry of Domestic Animals.(6a.ed., 932p.) Academic Press.

Kertz, A. F., Hill, T. M., Quigley, J. D., Heinrichs, A. J., Linn, J. G. \& Drackley, J. K. (2017). A 100-year review: calf nutrition and management. Journal of Dairy Science, 100, 10151-10172.

Knowles, T. G., Edwards, J. E., Bazeley, K. J., Brown, S. N., Butterworth, A. \& Warriss, P. D. (2000). Changes in the blood biochemical and hematological profile of neonatal calves with age. Veterinary Record, 174(21), 593-598.

Marçal, W. S., Birgel, E. H., D’angelino, J. L. \& Miguel, O. (1995). Avaliação cronológica da variação no hematócrito sanguíneo de bovinos leiteiros. Ciência Rural, 25(2), 239- 243.

Mitra, R., Adamec, R. \& Sapolsky, R. (2009). Resilience against predator stress and dendritic morphology of amygdala neurons. Behavioural Brain Research, 205, 535-543.

Muller, J. (2017). Dehydration as a risk factor for calf mortality in northern Australia. Masters thesis, University of Queensland. Recuperado de: https://espace.library.uq.edu.au/view/UQ:690280

Mohri, M., Sharifi, K. \& Eidi, S. (2007). Hematology and serum biochemistry of Holstein dairy calves: age related changes and comparison with blood composition in adults. Research Veterinary Science, 83, 30 - 39.

Nathanielsz, P. W., Lowe, K. C., Beck, N. F. G., Mcnaughton, D. C. \& Jansén, C. A. M. (1980). Circulation plasma protein concentrations in the fetal and neonatal sheep. Biology of Neonatal, 38, 126-133.

Novo, S. M. F., Freitas, R. L., Silva, C. P. C., Baldacim, V. A. P., Baccili, C. C., Reis, J. F., Hagiwara, M. K. \& Gomes, V. (2015). Hematological adaptation in Holstein calves during the neonatal period.Brazilian Journal of Veterinary Research and Animal Science, 52(3), 212-216.

Paes, P. R. O., Gonçalves, R. C., Barioni, G., Leme, F. O. P., Melo, M. M. \& Cruz, M. L. O. (2012). Leucograma como indicador de estresse no desmame e no transporte rodoviário de bovinos da raça Nelore. Sêmina:Ciências Agrárias, 33(1), 305-312.

Panousis, N., Siachos, N., Kitkas, G., Kalaitzakis, E., Kritsepi-Konstantinou, M. \& Valergakis, G. E. (2018). Hematology reference intervals for neonatal Holstein calves. Research in Veterinary Science, 118, 1-10. 
Paula Neto, J. B. P. (2004). Hemogramas de bovinos (Bostaurus) sadios da raça curraleiro de diferentes idades, machos e fêmeas, gestantes e não gestantes. Dissertação (Mestrado em Ciência Animal) - Escola de Veterinária, Universidade Federal de Goiás, Goiânia.

Peixoto, A. P. C., Costa, J. N., Kohayagawa, A., Takahira, R. K. \& Saito M. E. (2002). Hemograma e metabolismo oxidativo dos neutrófilos de bovinos da raça Holandesa preta e branca: influência dos fatores etários. Revista Brasileira de Saúde e Produção Animal, 3(1), 16-20.

Pereira A. S., Shitsuka D. M., Parreira F. J., \& Shitsuka R. (2018). Metodologia da pesquisa científica. UFSM. https://repositorio.ufsm.br/bitstream/handle/1/15824/Lic_Computacao_Metodologia-Pesquisa-Cientifica.pdf?sequence=1

Pinna, M. H., Botteon, R. C. C. M., Júnior, J. C. B. S., Botteon, P. T. L. \& Giácomo, Z. (2001).Valores hematológicos de bezerros leiteiros mestiços de zero a três meses de idade. Anais da XI Jornada de Iniciação Científica da UFRRJ.

Ramin, A,G., Asri-Rezaei, S., Paya, P., Eftekhari, Z., Jelodary, M., Akbari, H. \& Ramin, S. (1975). Evaluation of Anemia in Calves up to 4 Months of Age in Holstein Dairy Herds. Journal Vet Scan, 7(1), 87-92.

Renaud, D. L., Duffield, T. F., Leblanc, S. J. \& Kelton, D. F. (2018). Validation of methods for practically evaluating failed passive transfer of immunity in calves arriving at a veal facility. Journal of Dairy Science. 101(10), 9516-9520.

Rengifo, S. A., Silva, R. A., Botteon, R. C. C. M. \& Botteon P. T. L. (2010). Hemograma e bioquímica sérica auxiliar em bezerros mestiços neonatos e ocorrência de enfermidades. Arquivo Brasileiro de Medicina Veterinária e Zootecnia, 62(4), 993-997.

Rocha, T. G., Nociti, R. P., Sampaio, A. A. M. \& Fagliari, J. J. (2013). Hemograma e proteínas de fase aguda de bezerros sadios do nascimento aos 30 dias de idade. Pesquisa Veterinária Brasileira, 33, 25-31.

Schalm, O. W. (2010). Veterinary hematology. (2a ed., pp.307-3012). Lea and Febiger.

Souza, D. F., Kowalski, L. H., Kulik, C. H., Filho, I. R. B., Dittrich, R. L. \& Monteiro, A. L. G. (2018). Dinâmicas pré e pós-colostral do eritroleucograma, da proteína plasmática total e do fibrinogênio de cordeiros. Ciência Animal Brasileira, 19, 1-11 e 24805.

Távora, J.P. F. (1997). Hemograma de bovinos das raças Gir, Girolando e Holandesa criados no Estado de São Paulo - Estabelecimento dos valores de referência e avaliação das influências de fatores de variabilidade raciais, etários e sexuais. Tese (Doutorado em Clínica Veterinária) - Faculdade de Medicina Veterinária e Zootecnia, Universidade de São Paulo. São Paulo.

Taylor, J. A. (2000). Leukocyte responses in ruminants. In: Feldman, B.F., Zinkl, J.G. \& Jain, N.C. (ed.), Schalm's Veterinary Hematology. (5a ed., pp.391404). Lippincott Williams and Wilkins.

Tennant, B., Harrold, D. \& Reinaguerra, M. (1974). Haematology of the neonatal calf: Erithrocyte and leukocyte evalues of normal calves. Cornell Veterinarian, 64, 516-532.

Thrall, M. A., Weiser, G., Allison, R.W., \& Campbell, T. W. (2015). Hematologia e Bioquímica Clínica Veterinária. (2a.ed. 678p). Roca.

USDA.2016. Dairy 2014, Dairy Cattle Management Practices in EUA. USDA-APHIS: VS-CEAH-NAHMS, Fort Collins, CO.

Weiss, D. J. \& Wardrop, K. J. (2010). Schalm's veterinary hematology. (6a ed., 1206p) Wiley Blackwell.

Wickramasinghe, H. K. J. P., Kramera, A. J. \& Appuhamy, A. D. R. N. (2019). Drinking water intake of newborn dairy calves and its effects on feed intake, growth performance, health status, and nutrient digestibility. Journal of Dairy Science, 102(1), 377-387.

Wise, G. H., Caldwell, M. J., Parrish, D. B., Flipse, R. J. \& Hughes, J. S. (1947). Changes in cell volume and concentration of hemoglobin and of several inorganic constituents of the blood of calves during early postnatal development. Journal Dairy Science, 30, 983-993. 